\title{
Sipāh-i Ispahān : devenir des groupes consonantiques initiaux moyen-perses en persan
}

\author{
Agnès Lenepveu-Hotz
}

\section{INTRODUCTION}

Dans les mots où le moyen perse présente des groupes consonantiques initiaux, le persan contemporain n'a plus cette structure. On s'interrogera sur les raisons et l'époque de cette transformation. Lorsqu'on observe de près le lexique du persan des $\mathrm{X}^{\mathrm{e}}-\mathrm{XI}^{\mathrm{e}}$ siècles, on a rapidement une impression d'anarchie quant à la place de la voyelle ajoutée. Et ce flou est d'autant plus grand que pour certains mots, il existe des doublets, tantôt avec prothèse, tantôt avec anaptyxe : sipāh comme Ispahān ont la même origine moyen-perse, spāh, « armée $»^{1}$.

Le moyen perse manichéen et le judéo-persan permettent d'éclaircir ce qui paraît être un flottement. Personne n'ignore tout ce que l'on doit à Gilbert Lazard pour la compréhension des débuts du persan ${ }^{2}$ et du judéo-persan. C'est donc modestement que, m'appuyant sur les travaux de ce spécialiste incontesté, je tenterai de résoudre cette question du devenir des groupes consonantiques initiaux moyen-perses en persan.

Après avoir établi si une voyelle brise bien tous les groupes consonantiques initiaux dès les premiers textes persans, je me pencherai sur la place et le timbre de cette voyelle. La chronologie relative de ces changements révélera alors que l'alternative prothèse-anaptyxe est le fruit d'évolutions successives.

La présente analyse porte sur le développement des voyelles de prothèse et d'anaptyxe, de fait j'écarte la syllabe initiale $x w$ - - elle relève d'une autre question

\footnotetext{
${ }^{1}$ Les mots moyen-perses de cet article sont tirés du dictionnaire de MacKenzie (1970), pour le moyen perse manichéen, de ceux de Boyce (1977) et de Durkin-Meisterernst (2004). La transcription habituelle du moyen perse et du persan a ici été respectée, même lorsqu'elle ne suit pas l'Alphabet Phonétique International (Voir le système dans Mackenzie 1970 et dans Lazard 1963).

${ }^{2}$ Pour plus de lisibilité, «persan » correspond à l'état de langue noté en caractères arabo-persans. Abréviations utilisées : angl. : anglais ; C : consonne ; fr. : français ; m.p. : moyen perse ; pn. : persan ; $\mathrm{V}$ : voyelle.
} 
phonologique -, ainsi que les phénomènes d'interversion, par exemple le moyen-perse frazand, « enfant », devenu en persan farzand.

\section{PERSISTANCE DE GROUPES CONSONANTIQUES INITIAUX ?}

Le fait que les groupes consonantiques initiaux n'existent plus en persan contemporain ne suppose pas nécessairement que tel soit déjà le cas en persan ou en judéo-persan des $\mathrm{X}^{\mathrm{e}}-\mathrm{XI}^{\mathrm{e}}$ siècles.

\subsection{Deux écritures ambigües}

Si le problème se pose, c'est parce que tant le persan que le judéo-persan sont écrits dans des écritures adaptées aux langues sémitiques et à leur structure, qui de ce fait ne notent pas les voyelles brèves. Ainsi br'dr, translittération ${ }^{3}$ commune du persan بر ادر et du judéo-persan בראדר, est-il à lire barādar comme en persan contemporain (ou plutôt birādar) ou bien comme le brādar du moyen perse ? Comment alors vérifier que dès les $\mathrm{X}^{\mathrm{e}}-\mathrm{XI}^{\mathrm{e}}$ siècles, la voyelle d'anaptyxe $\mathrm{e}^{4}$ est déjà apparue ? Poser la question pour le judéo-persan est d'autant plus légitime que, comme Lazard l'a montré à de nombreuses reprises ${ }^{5}$, il s'agit d'une langue très proche du moyen perse et que leurs différences ne sont dues qu'à

« ce qui distingue ordinairement une langue littéraire d'une langue parlée courante $»^{6}$.

Pour d'autres faits d'évolution phonologique entre le moyen perse et le persan, l'arménien apporte habituellement des éclairages pertinents. Ici il n'est toutefois d'aucun secours : Pisowicz ${ }^{7}$ constate que l'arménien omet régulièrement de noter

\footnotetext{
${ }^{3}$ Afin de les distinguer, la translittération est écrite en caractères dits « romains », la transcription en italique.

${ }^{4}$ Les phénomènes de prothèse, eux, sont bien entendu clairement notés dans les deux écritures, avec la présence d'un alef ( $\mathrm{l}$ et $\mathrm{\kappa}$ ).

${ }^{5}$ En dernier lieu dans Lazard 2003.

${ }^{6}$ Lazard 2003, p. 100.

${ }^{7}$ Pisowicz 1985, pp. 78 et 128.
} 
l'anaptyxe dans les groupes consonantiques initiaux. Il ne nous reste donc que le recours aux textes persans eux-mêmes.

\subsection{Cas du persan}

Pour le persan, confirmer la présence de voyelles d'anaptyxe est chose facile. La valeur métrique d'un mot contenant cette voyelle diffère de celle du mot sans cette voyelle. La scansion d'un vers, de Firdausī par exemple (1), permet de lever le doute sur la lecture à avoir de xrd, « raison ». Ce mot est situé ici en début de mesrā ` et, dans le vers propre à l'épopée, le premier pied se compose obligatoirement d'une syllabe brève suivie de deux longues. Si le mot s'était encore prononcé xrad comme en moyen perse, avec ici le suffixe -mand, nous aurions eu deux longues $(--)^{8}$. Avec xirad-mand, le vers débute bien par une brève suivie de deux longues conformément au mètre choisi $(\mathrm{u}--)$.

(1) $\mathrm{u}--|\mathrm{u}--| \mathrm{u}--\mid \mathrm{u}-$ xiradmand-u hamčūn pidar nāmjōy

« sage et à la recherche de la gloire comme (son) père » (Šāhnāma, Gayōmart, v. 14, 2nd mesrā')

Dans son étude de phonologie quantitative sur le Šāhnāma, Moinfar lit xirad (1973, p. 31, 1. 55, p. 46, 1. 434), avec voyelle d'anaptyxe. Il en fait de même pour d'autres mots tels que sipéd, «blanc » (p. 33, 1. 111) ; farmūd, « ordonna » (p. 35, 1. 189); girift, « prit » (p. 38, 1. 291) ; sarāy, «maison » (p. 40, 1. 345), etc.

Certains cas de vocalisation dans les manuscrits attestent d'autre part de cette présence de la voyelle d'anaptyxe dès les premiers siècles. Lazard ${ }^{9}$ en donne des exemples dont daraxt, «arbre », sapēd, «blanc », farōd āyad, « descend ». Et comme il le souligne, ces vocalisations émanent essentiellement de deux textes, conservés dans deux des manuscrits les plus anciens dont nous disposons : le Kitāb al-abniya

\footnotetext{
${ }^{8}$ C'est avec une longue que se scande $x r a d$ dans un poème moyen-perse analysé par Lazard (2002, p. 132).

${ }^{9}$ Lazard 1963, pp. 187-188, § 137.
} 
d'Abū Mansūr Muvaffaq (manuscrit de Vienne daté de 447/1056, le plus ancien manuscrit persan) et le Kitāb hidāyat al-muta 'allimīn fì al-tibb d'Abū Bakr Rabī' b. Ahmad al-Axavain̄̄ al-Buxārī (manuscrit de la Bibliothèque Bodléienne de 478/1085). Les voyelles d'anaptyxe sont donc clairement attestées dès le $\mathrm{XI}^{\mathrm{e}}$ siècle par ces manuscrits, peut-être même dès le $\mathrm{X}^{\mathrm{e}}$ siècle, date de la composition de ces ouvrages.

Cependant le grammairien persan Šams-i Qais ${ }^{10}$ affirme que dans certains dialectes, les groupes consonantiques initiaux existent. On constate ce phénomène dans le Codex Cumanicus $^{11}$, et encore de nos jours dans la langue parlée au Sistān ${ }^{12}$. Mais en observant ces mots à groupe consonantique initial, on s'aperçoit que si certains ont bien la même structure que le moyen perse, d'autres présentent un schéma $\mathrm{CC}$ - là où le moyen perse avait CVC-. Ainsi pour le Codex Cumanicus $^{13}$ : draxt, «arbre », frāmū̌s « oubli », comme en moyen perse mais drūgār, « charpentier» (m.p. durgar), grīxdan, « fuir » (m.p. wirêxtan), psar, «fils » (m.p. pus), etc. Il en est de même pour la langue du $\operatorname{Sistān}^{14}$ : frōš/froxt, « vendre », gir/graft, «prendre », et škan/škast, « briser », avec un groupe CC- identique au moyen perse, à côté de $k \check{s}^{-}$, «tuer » (m.p. kuštan/kuš-), nšin/šnast, «s'asseoir » (m.p. nišastan/nišīn), nvis/nvešt, « écrire » (m.p. nibištan/nibess), et tn (tr)/tmest (trest), « pouvoir» (m.p. tuwān), tandis que le moyen perse avait une structure CVC-. Il faut donc supposer que ces groupes sont dus à un développement ultérieur et dialectal, et qu'ils ne remettent pas en cause l'apparition de voyelle d'anaptyxe à époque ancienne. En effet Šams-i Qais écrit aux XII ${ }^{\mathrm{e}}-\mathrm{XIII}{ }^{\mathrm{e}}$ siècles et le Codex Cumanicus date du $\mathrm{XIV}^{\mathrm{e}}$ siècle alors que vocalisations dans des manuscrits et poésie des $\mathrm{X}^{\mathrm{e}}-\mathrm{XI}^{\mathrm{e}}$ siècles prouvent la présence de voyelles d'anaptyxe.

\subsection{Situation en judéo-persan}

\footnotetext{
${ }^{10}$ Grammairien des XII $^{\mathrm{e}}-\mathrm{XIII}{ }^{\mathrm{e}}$ siècles, cité par Sādeghi (2001, p. 18).

${ }^{11}$ C'est ce que donne Pisowicz (1985, p. 128) comme exemple de maintien des groupes consonantiques initiaux en persan. Pour mémoire, le Codex Cumanicus est un lexique latin-turc-persan en alphabet latin, compilé dans la première moitié du XIV ${ }^{\mathrm{e}}$ siècle.

12 Lazard 1974, p. 66.

${ }^{13}$ Bodrogligeti 1971, pp. 61-62.

${ }^{14}$ Lazard 1974, pp. 84-85.
} 
Si le persan ne présente pas de groupes consonantiques initiaux, qu'en est-il du judéo-persan ${ }^{15}$ ? De nouveau le problème de l'écriture se pose, mais accru par la présence d'un signe diacritique, le shewa. Pour notre corpus, on le trouve uniquement dans le Tafsīr d'Ezéchiel (TE). Il y est ambigu parce qu'il note à la fois l'absence de voyelle entre deux consonnes (comme le sokun arabe) et la présence d'une voyelle brève, sans indication du timbre. Gindin ${ }^{16}$ illustre ce second phénomène : pour noter un /ă/ dans $\mathrm{d}^{\overline{\mathrm{o}}} \mathrm{wk}^{\partial} \mathrm{r}^{\mathrm{a} h}$ (avec kardan, «mentionner ») (TE 1, 24), à comparer avec $\mathrm{d}^{\overline{0}} \mathrm{wk}^{\mathrm{a}} \mathrm{r}^{\mathrm{a}} \mathrm{h}(\mathrm{TE} 1,30)$; un / $\mathbf{1} /$ dans $\mathrm{k}^{2} \mathrm{l}^{\mathrm{e}} \mathrm{yp}(\mathrm{TE} 5,4)$ vs. $\mathrm{k}^{\mathrm{i}} \mathrm{l}^{\mathrm{e}} \mathrm{yp}$ (TE 5, 7), « opposition »; un $/ \breve{\mathrm{u}} /$ dans $\mathrm{m}^{\ominus} \mathrm{r}^{\overline{\mathrm{a}}} \mathrm{d}^{\partial} \mathrm{g}^{\mathrm{i}} \mathrm{yn}$ (TE 20, 11) vs. mwr'dgyn (TE 20, 13), « satisfait ». Dans $m^{\ominus} r^{\bar{a}} d^{\ominus} g^{i} y n$, signalons, en comparant les deux graphies de ce mot, que le premier shewa a la même valeur que le w, mater lectionis d'un / $\breve{\mathrm{u}} /$ tandis que le second indique une absence de voyelle (ajout du suffixe -gyn à mwr'd).

Mais alors, quand le shewa apparait dans un groupe consonantique initial, comment savoir s'il correspond à une absence de voyelle ou bien à une voyelle brève d'anaptyxe ? Dans le premier cas, nous aurions une structure CC- comme en moyen perse ; rappelons la proximité du judéo-persan et du moyen perse - proximité ne voulant néanmoins pas dire identité, d'autant plus que cette différence entre les deux relève de la distinction littéraire/parlé ${ }^{17}$. Dans le second, ce serait le schéma CVC-, à l'instar du persan. Ainsi $b^{\partial} r^{\bar{e}} y h$, «splendeur » (TE 64, 22), est-il à lire comme le moyen-perse brāh ou bien comme le persan barāh? Le changement de timbre vocalique de la voyelle finale $/ \bar{a} /$ en $/ \bar{e} /$ laisserait supposer qu'est survenu un phénomène de palatalisation de ce $/ \bar{a} /$ au contact d'une voyelle palatale ${ }^{18}$ : il faudrait

\footnotetext{
${ }^{15}$ Voici les textes judéo-persans utilisés ici : Ahv. : document juridique de Ahvāz (1020), édité par Asmussen (1965); DU : lettre découverte à Dandān-Uiliq (édition d'Utas en 1969); EA : fragment d'apologétique, publié par MacKenzie (1968) ; GK : texte grammatical karaïte (Khan 2000) ; L : lettres, étudiées par Paul (Je remercie Ludwig Paul pour ces documents.) ; TE : Tafsìr d'Ezéchiel, publié par Gindin (2007).

${ }^{16}$ Gindin 2007, III, Orthography, § 3.3. Thamar Gindin m'a librement donné accès à son travail, qu'elle en soit ici remerciée.

${ }^{17}$ On peut comparer cela au français où la prononciation méridionale de /pənø/ correspond à pneu dans l'écriture (et dans la prononciation d'autres régions).

${ }^{18}$ Nous pouvons comparer ce phénomène à celui de l'imāla dans les mots d'origine arabe (Gindin 2007, III, Phonology, § 1.2.1).
} 
alors attribuer ce timbre au shewa et lire birē $h^{19}$. Mais rien de définitif ne permet de valider cette hypothèse.

Des occurrences dotées d'une vocalisation autre que le shewa pourraient lever cette ambiguïté. Les deux seuls cas dont nous disposons ${ }^{20}, \mathrm{~g}^{\overline{\mathrm{e}}} \mathrm{r}^{\mathrm{\partial}} \mathrm{y}^{\bar{a}}, \overline{\mathrm{a}} \mathrm{n}$, « ceux qui pleurent » (TE 32, 9), et g ${ }^{\bar{e}} \mathrm{r}^{\overline{1}} y^{\mathrm{a}} \mathrm{d}$, « il pleure » (TE 135, 9), apparaissent cependant dans le premier dialecte du Tafsīr d'Ezéchiel, qui émane du nord-ouest ${ }^{21}$. Ce texte est en effet écrit dans deux dialectes bien distincts : $\mathrm{TE}_{1}$, caractéristique du nord et $\mathrm{TE}_{2}$, proche des autres textes judéo-persans, du sud-ouest ${ }^{22}$. Or les shewa aussi ne se rencontrent que dans ce dialecte du nord.

Afin de déceler l'éventuelle présence de voyelles d'anaptyxe en judéo-persan, il reste néanmoins un dernier type d'indices à exploiter, les cas de mater lectionis : une voyelle brève notée avec le signe consonantique habituellement utilisé pour indiquer une longue (alef א pour /a/, yod' pour /i/ et vav ı pour/u/). Par exemple bwrhn', «nu » (TE 62, 1 et 82, 20). De nouveau $\mathrm{TE}_{2}$ n'en présente pas. Quant aux autres textes (Ahv., $\mathrm{EA}, \mathrm{GK}$ et L) ${ }^{23}$, seul L4 contient une mater lectionis, dans le verbe « envoyer », et ce pour toutes ses occurrences : pyrystynd (L4, 6) ; pyrystym (L4, 7 et 17$)^{24}$. Cette lettre commerciale aurait été rédigée à Basrah ${ }^{25}$, autrement dit dans le sud-ouest du domaine iranien, tout comme les autres textes cités. Il est donc inattendu d'y voir une mater lectionis, isolée dans ces dialectes judéo-persans du sud-ouest.

19 On trouve d'autres exemples de vocalisation avec un shewa comme $\mathrm{d}^{\mathrm{a}} \mathrm{r}^{\mathrm{a}} \mathrm{m}^{\overline{ }} \mathrm{yh}^{\overline{\mathrm{a}}}$, « drachmes » (TE

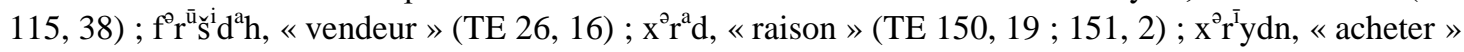
(TE 26,$12 ; 26,15)$.

${ }^{20}$ Gindin (2007, III, Phonology, § 1.4) cite aussi $d^{a} r^{\overline{0}} \mathrm{wd}^{\mathrm{i}} \mathrm{yh}^{\mathrm{a}}$, « , planche » (TE 76, 27). Mais ce mot est certainement lié aux moyen-perses dāru, «arbre », dārūg, «plante », donnés par Boyce (1977). Ici il n'y aurait donc pas de voyelle d'anaptyxe mais une voyelle étymologique.

21 Sur cette localisation, voir les arguments de Lazard (à paraître). Je remercie vivement Monsieur Lazard de m'avoir communiqué cet article.

${ }^{22}$ Voici leur répartition dans le manuscrit (Gindin 2007, I, p. 23) : TE 1 pp. 1-169 et 221-226; TE 2 pp. 170-220.

${ }^{23}$ Je laisse de côté les formes du verbe « couper » en bwryd- puisque dès le moyen perse, burìdan est en concurrence avec brīdan (Cf. Mackenzie 1970).

${ }^{24}$ Un autre cas suggèrerait la présence d'une voyelle intermédiaire : pyrm'n, « ordre » (Ahv. 1. 4), qui est à rapprocher du persan farmān et non du moyen-perse framān. Mais il ne s'agit pas d'une voyelle d'anaptyxe, c'est un cas d'interversion.

${ }^{25}$ Cf. remarque de Paul (2002). 
Les occurrences de shewa, de vocalisation et de mater lectionis se rencontrent toutes, à une exception près, dans le premier dialecte du Tafsīr d'Ezéchiel. Les textes caractéristiques du sud-ouest en sont presque totalement dépourvus. Cela supposeraitil que les groupes consonantiques initiaux du moyen perse étaient encore préservés dans le judéo-persan du sud-ouest? On pourrait le croire. Mais comme aucun de ces signes n'est obligatoire - ce n'est qu'une possibilité laissée au copiste -, on ne peut déduire de leur absence que ces groupes ont été préservés; la majorité des textes persans en écriture arabo-persane ne présente pas non plus de vocalisation indiquant une voyelle d'anaptyxe, voyelle pourtant attestée dans d'autres textes ou dans la poésie $^{26}$.

\section{AJOUT D'UN PHONEME VOCALIQUE}

Bien que l'ajout de cette voyelle ne soit pas clairement établi dans tous les dialectes, le problème persiste : connaître la place et le timbre de cette éventuelle voyelle brisant le groupe consonantique initial du moyen perse. Répondre à ces questions permettra peut-être de nous renseigner sur le processus de disparition de ces groupes consonantiques.

\subsection{Prothèse ou anaptyxe?}

L'alternative prothèse-anaptyxe est-elle bien liée, comme le croit Lentz $^{27}$, à des différences dialectales, avec anaptyxe dans le sud-ouest et prothèse au nord ? Rien n'est moins sûr. Cette idée est bousculée par de nombreuses occurrences. Des mots se rencontrent uniquement avec anaptyxe, au nord comme au sud-ouest (firistādan et pyryst dans L4), tandis que d'autres connaissent les deux solutions, et ce dans les deux régions. Ainsi en judéo-persan le verbe šināxtan, «connaître »: ’šnxtn (EA N1) et šnxtn (EA T11); en persan istadan et sitadan, «prendre $»^{28}$. Cette répartition n'est donc pas d'ordre géographique.

\footnotetext{
${ }^{26}$ Les textes judéo-persans des $\mathrm{X}^{\mathrm{e}}-\mathrm{XI}^{\mathrm{e}}$ siècles sont en prose.

${ }^{27}$ Lentz 1926, pp. 294 et 308-309.

${ }^{28}$ Cf. Lazard 1963, p. 175, § 105.
} 
Pour comprendre dans quels cas la voyelle ajoutée est une prothèse et dans lesquels c'est une voyelle d'anaptyxe, il faut partir des groupes consonantiques initiaux existant en moyen perse. On rencontre les possibilités suivantes : br- ; dr- ; tr- ; gr- ; fr- ; sr- ; zr- ; xr- ; dw- ; $\mathrm{xw}^{-29}$ ainsi que sp- ; st- ; sk- ; šk-; šn-. Tous ces groupes se répartissent en $\mathrm{C}-\mathrm{r}, \mathrm{C}-\mathrm{w}, \mathrm{s}-\mathrm{C}, \mathrm{s}$ - $\mathrm{C}$. Dans les deux premières structures, $\mathrm{C}$ peut être une occlusive ou une spirante; dans les deux autres, C est exclusivement une occlusive. Voici le devenir de ces groupes consonantiques en persan, parfois avec anaptyxe, parfois avec prothèse ou anaptyxe :

\begin{tabular}{|c|c|}
\hline Anaptyxe & Prothèse ou anaptyxe \\
\hline br-> bir- / bar- / bur- & $\begin{array}{l}\text { sp- > sip- / sap- } \\
\text { sp- > isp- }\end{array}$ \\
\hline dr- > dir- / dar- / dur- & $\begin{array}{l}\text { st- > sit- / sut- } \\
\text { st- > ist- }\end{array}$ \\
\hline$(\operatorname{tr}->\operatorname{tur}-)^{30}$ & $\begin{array}{l}(\mathrm{sk}->\text { sik- } \\
\text { sk- > isk- })^{31}\end{array}$ \\
\hline gr- > gir- / gur- & $\begin{array}{l}\text { šk- > šik- / šig- / šuk- } \\
\text { šk- > ašk- }\end{array}$ \\
\hline fr- > far- / fir- / fur- & $\begin{array}{l}\text { šn- > šin- / šun- } \\
\text { šn- > ašn- }\end{array}$ \\
\hline sr- > sar- / sir- / sur- & \\
\hline$(\text { zr- > zir- })^{32}$ & \\
\hline xr- > xir- / xar- / xur- & \\
\hline$(\mathrm{dw}->\text { duv- / dav- })^{33}$ & \\
\hline
\end{tabular}

${ }^{29}$ Je ne parlerai pas du devenir de xw- en persan car il n'est pas lié au problème de prothèse et d'anaptyxe.

${ }^{30}$ La sourde tr-, déjà peu représentée en moyen perse, ne s'est conservée que dans un mot persan turš, avec interversion (m.p. $\operatorname{tru}(f)$ š).

${ }^{31}$ Cela ne correspond qu'à un unique mot : sikandar / iskandar, « Alexandre ».

${ }^{32}$ Pour zr-, un mot conservé en persan : zrēh > zirih, «armure».

${ }^{33}$ Il est difficile de tirer une règle pour ce groupe, représenté par le seul mot $d w \bar{a} z d a h$, «douze » > duvāzdah. 
Le développement des voyelles semble suivre la règle suivante :

- une voyelle d'anaptyxe dans les groupes C-r

- une voyelle de prothèse ou une voyelle d'anaptyxe pour les groupes $\mathrm{s}-\mathrm{C}$ et š-C où $\mathrm{C}$ est une occlusive.

$\mathrm{Au}$ vu de ces résultats, on ne peut affirmer, comme le fait Sādeghi ${ }^{34}$, que c'est la première consonne qui importe, autrement dit qu'un groupe débutant par une occlusive est brisé par une voyelle d'anaptyxe, tandis qu'un groupe avec spirante à l'initiale l'est avec prothèse ou anaptyxe. Au contraire, étant donné que les spirantes se retrouvent dans les deux colonnes, ce qui importe c'est la nature de la seconde consonne : /r/ avec voyelle d'anaptyxe; occlusive avec voyelle de prothèse ou d'anaptyxe.

Il existe néanmoins quelques cas avec prothèse dans des groupes C-r :

- br- avec une prothèse dans $a b r \bar{u}$, «sourcil »: doit-on voir avec Lentz ${ }^{35}$ un mot emprunté à un dialecte du nord (parthe ?) à côté de barū? Mais dans le parthe des textes manichéens, Boyce (1977) et Durkin-Meisterernst (2004) ne relèvent aucune occurrence de ce mot.

- l'hésitation entre prothèse et interversion pour m.p. srub, «plomb»> pn. usrub (اسرب) / surb ${ }^{36}$. On ne trouve pas d'occurrence de ce mot avec anaptyxe.

- 2 mots avec prothèse en judéo-persan : dans le Tafsīr d'Ezéchiel, 'krd pour xirad, « raison » (TE 150, $22 ; 150,30 ; 153,29 ; 154,5)$; dans plusieurs textes judéopersans (dont les deux dialectes de TE), 'prwd pour le préverbe furōd, « vers le bas ». Pour le premier mot, la situation avec anaptyxe existe aussi. Pour le second, Paul ${ }^{37}$ y lit la préposition $u$ suivie de furōd, parallèlement à des formes comme 'pyš ( $\bar{o}-p \bar{e} \check{s}$, «avant»). Contre cette interprétation, Gindin ${ }^{38}$ avance que les autres prépositions combinées avec $\bar{o}$ apparaissent également utilisées seules mais que furōd est toujours

\footnotetext{
${ }^{34}$ Sādeghi 1970, p. 544 et 2001, p. 17.

${ }^{35}$ Lentz 1926, p. 274.

${ }^{36}$ Cf. Lazard 1963, p. 175, § 105.

${ }^{37}$ Paul 2003, p. 183.

${ }^{38}$ Gindin 2007, III, Phonology, § 1.5.
} 
précédé de cet alef, dans les deux dialectes de TE. Or Lazard ${ }^{39}$ remarque que la préposition $u$ (à lire en fait $a$ ) n'est jamais employée dans le premier dialecte de TE.

Ces exceptions sont toutefois trop peu nombreuses pour remettre en cause le schéma général. D'ailleurs, même si les lois phonétiques ne sont valables que pour une époque donnée, on retrouve la même répartition entre anaptyxe et prothèse dans les mots étrangers empruntés par le persan contemporain : anaptyxe avec $/ \mathrm{r} /$ en seconde consonne (teran < fr. train ; šerāpnel < angl. shrapnel ; Seri Lānkā pour Sri Lanka) et prothèse avec occlusive en seconde consonne (eski, esport < fr. ski, angl. sport). Cependant en persan contemporain, il n'existe pas d'hésitation entre anaptyxe et prothèse pour les groupes avec occlusive comme seconde consonne. J'y reviendrai ${ }^{40}$. Retenons pour le moment que ce même fonctionnement témoigne peut-être d'un lien avec les sons eux-mêmes et d'une facilité de syllabation. Karimi ${ }^{41}$ constate que l'espagnol a développé une stratégie identique; elle l'attribue au fait que /s/ en fin de la première syllabe initiale VC- serait moins marqué que les autres consonnes, occlusives ou autres fricatives que $/ \mathrm{s} /{ }^{42}$.

\subsection{Timbre de la voyelle ajoutée}

Sādeghi ${ }^{43}$ présente un tableau très clair du timbre de la voyelle d'anaptyxe et de prothèse pour les groupes à occlusive en seconde position :

- /i/ dans la majorité des cas

- /u/ quand le mot comporte une seconde voyelle, de timbre $/ \overline{\mathbf{o}} /, / \overline{\mathrm{u}} /, \mathrm{l} / \mathrm{u}$.

Il donne quelques exceptions à la règle qu'il propose, telles que astān/ustān, «province », sarā/surāa, «maison »; šikūfa, «fleur », šimurdan, « compter ». Il précise aussi que l'on trouve d'autres vocalisations dans certains textes ou dans des

\footnotetext{
${ }^{39}$ Lazard 2009, p. 173.

${ }^{40}$ Cf. infra, $\$ 4.3$.

${ }^{41}$ Karimi 1987, p. 314.

42 L'explication de Sādeghi (2001, p. 19) sur le fait que la syllabe la plus "agréable" et la plus "recherchée" serait de structure CV- est ainsi contredite par les exemples persans comme espagnols.

${ }^{43}$ Sādeghi 2001, pp. 19-20. Curieusement il ne parle pas des voyelles d'anaptyxe dans les groupes Occlusive-/r/. En outre il ne distingue pas dans ses relevés les cas où la prothèse est réelle $\left({ }^{\mathrm{v}} \mathrm{CC}\right)$ de ceux où la voyelle existe dès le moyen perse (VCC).
} 
dialectes. En réalité, aux exceptions qu'il relève s'en ajoutent beaucoup d'autres, à tel point que l'on est presque amené à se demander si la règle qu'il présente en est vraiment une. Il s'agirait moins d'une règle que d'une tendance. De plus il faudrait ajouter que la voyelle d'anaptyxe est souvent /a/ quand le mot comporte une seconde voyelle, de timbre /a/ ou /ā/. Ce /a/ est employé en concurrence avec /i/ pour certains mots (daraxt, «arbre », daram, «diram», etc. ${ }^{44}$ ), ou comme unique voyelle d'anaptyxe (barahna, «nu », farāmōš, « oubli », etc.). Dans le premier cas, ces mots sont ainsi vocalisés dans les deux manuscrits très anciens déjà évoqués : on aurait là le reflet, peut-être dialectal, de l'hésitation sur le timbre de la voyelle ajoutée ${ }^{45}$.

Cependant, de nouveau, il n'est ici question que d'une tendance puisqu'on rencontre

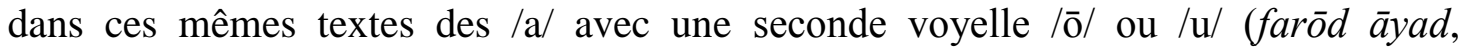
«descend », šapuš, «pou », garōhe, «groupe ») ${ }^{46}$. Au vu des exemples donnés par Lazard dans ces deux manuscrits, on a la nette impression que la voyelle privilégiée est le /a/, face au /i/ que Sādeghi relève dans les autres textes.

Quant au rôle que pourraient avoir les consonnes sur ce timbre vocalique, la seule trace se trouve dans le mot duvāzdah, «douze» (m.p. dwāzdah), où le /v/ a probablement influencé le /u/. Le judéo-persan apporte un autre terme à l'appui de cette hypothèse : dwrwyš, "pauvre », pour le darvēš du persan, même s'il s'agit ici d'interversion et non d'anaptyxe (m.p. driyōš).

Ce qui paraît en fait le plus certain, c'est que la voyelle brève ajoutée, comme d'autres voyelles brèves d'ailleurs, est instable. C'est encore le cas en persan contemporain $^{47}$ (par exemple setudan et sotudan, « louer »). Le shewa du judéo-persan en témoigne, il est probablement à lire comme une voyelle intermédiaire entre /a/ et /i/, peut-être comme un $/ \partial / /^{48}$. La voyelle ajoutée dans les premiers temps pourrait donc être cette voyelle centrale, tant sur l'aperture que sur le point d'articulation. Cette

${ }^{44}$ Tirés de Lazard 1963, pp. 187-188, § 137.

${ }^{45}$ Cf. supra, $\S 2.2$.

${ }^{46}$ Mots cités par Lazard (1963, pp. 187-188, § 137).

${ }^{47}$ Voir Lazard 2006, p. 12, § 7.

${ }^{48}$ Voir Sādeghi 1970, p. 549, repris par Pisowicz 1985, p. 127 ; Sādeghi 2001, p. 18. Nous avons vu au $\S 2.3$ que selon les mots /a/ alternait avec une vocalisation en /a/, en /i/, en /u/. 
voyelle n'existant pas ailleurs en persan, elle serait devenue, selon des règles plus ou moins suivies, tantôt /a/, tantôt /i/, tantôt /u/.

\section{LES PHASES DE L'EVOLUTION}

A ne regarder que le moyen perse et le persan, on pourrait croire que le développement des voyelles de prothèse et celui des voyelles d'anaptyxe se sont produits simultanément. Il n'en est rien. L'étude du moyen perse manichéen et du judéo-persan permet d'établir une chronologie relative, peut-être même des hypothèses sur les causes ${ }^{49}$ de ces évolutions.

\subsection{Prothèse}

Selon Thomason ${ }^{50}$, la disparition des groupes consonantiques initiaux en comox est due à influence du kwakiutl. Pour le persan, cette disparition est-elle due aussi à un facteur externe ? L'arabe a-t-il eu un rôle particulier, comme il en a eu un pour l'évolution de /p/ à /f/ dans certains vocables ? En réalité, l'évolution est antérieure aux contacts du persan avec l'arabe, elle remonte au moyen perse manichéen (textes et inscriptions datant des $\mathrm{III}^{\mathrm{e}}$ aux $\mathrm{X}^{\mathrm{e}}$ siècles). Si les groupes $\mathrm{C}-\mathrm{r}$ y sont bien conservés, les groupes C-Occlusive, eux, ont déjà tous connu l'ajout d'une voyelle prothétique :

- sp- : 'spr, « bouclier»

- st- : 'stwn, « colonne»

- šk- : ‘škyft, « sévère »

- šn- : 'šn's-/‘šn's-, ‘šn’xtn, « connaître $»^{51}$.

On sait par ailleurs que le moyen perse manichéen note la prononciation en usage à l'époque là où le moyen perse des livres use de graphies historiques ou pseudohistoriques. On peut alors se demander si l'évolution a eu lieu en moyen perse

\footnotetext{
${ }^{49}$ Sādeghi (2001, p. 16) annonce cette recherche des causes comme troisième point de son étude mais hélas, elle prend fin à la deuxième partie, du moins dans ce qui a été publié.

${ }^{50}$ Thomason 2001, p. 1643.

51 Pour d'autres exemples, voir Boyce 1977 et Durkin-Meisterernst 2004. Voir aussi dans le Šābuhragān, texte du III $^{\mathrm{e}}$ siècle (MacKenzie 1979-1980) : 'stptyh (p. 514, 1. 264'); 'stpt (p. 518, 1. 330); 'st(m)[b](g) (p. 518, 1. 343).
} 
également, mais sans être notée ? Pisowicz ${ }^{52}$ montre toutefois que ce n'est pas le cas au III $^{\mathrm{e}}$ siècle, au vu des transcriptions grecques telles que $\sigma \pi \alpha \pi \imath \tau$ ov face au moyenperse sp'hpt, «général ». Plus tardivement, on trouve encore stāyīdag, « loué », où l'absence de prothèse se voit confirmée par la métrique ${ }^{53}$.

Pour quelle raison ces groupes ont-ils été brisés par une voyelle de prothèse plus que par une voyelle d'anaptyxe? S'agirait-il d'une analogie sur d'autres mots moyenperses ? Cela est envisageable. En effet il existe des mots en asp-, ast-, ašk-, ašn- ; mais il n'existe aucun mot en sVp-, sVt-, et peu en šVk- (̌̌akar, « sucre ») et en šVn$\left(\check{s} a n\right.$, «chanvre $\left.{ }^{54}\right)$. Afin d'éviter les groupes consonantiques initiaux, les locuteurs auraient donc eu recours à une structure de la langue déjà existante ${ }^{55}$.

La différence de traitement entre les deux types de groupes consonantiques est en définitive d'ordre temporel. S'il y a hésitation entre prothèse et anaptyxe pour les groupes C-Occlusive, c'est parce que ces groupes avaient évolué vers une structure VCC dès le moyen perse manichéen et que, à un moment donné, cette prothèse s'est trouvée en concurrence avec une voyelle d'anaptyxe, elle, plus récente.

\subsection{Anaptyxe en persan et judéo-persan}

Pour les groupes C-r, nous avons établi que, à de très rares exceptions, c'est une voyelle d'anaptyxe qui a été ajoutée. Ces groupes se sont maintenus en moyen perse manichéen (par exemple brādar, draxt, grift) et la voyelle d'anaptyxe date des débuts du persan. De plus il n'est pas assuré que ces groupes n'existaient pas encore en judéopersan, du moins dans le judéo-persan du sud-ouest. Il en résulte que le développement de la voyelle d'anaptyxe dans les groupes C-r est plus tardif que celui de la voyelle de prothèse dans les groupes C-Occlusive.

L'apparition de ces voyelles d'anaptyxe en persan peut-elle alors être due à un facteur externe? L'arabe a pu vraisemblablement jouer un rôle de catalyseur,

\footnotetext{
52 Pisowicz 1985, p. 147.

${ }^{53}$ Voir Lazard 2002, p. 132, v. 3 et son analyse métrique p. 134. Notons aussi l'absence d'anaptyxe dans les groupes C-r (grift et $x \mathrm{rad}$ ) de ce même texte.

${ }^{54} \mathrm{Un}$ autre mot existe mais avec une voyelle longue.

55 Nous avons vu (§ 3.1) que pouvait s'y ajouter un argument de facilité de la syllabation avec une structure VsC.
} 
davantage peut-être que celui de déclencheur. On pourrait également songer à une influence du parthe. Ainsi selon $\operatorname{Paul}^{56}$, le persan durō $\gamma$, «mensonge », est à rapprocher du parthe $d u r \bar{o} \gamma$, par contraste avec le moyen-perse $d r \bar{o}(w)^{57}$. Mais il s'agit de cas isolés ${ }^{58}$ en regard des nombreux groupes initiaux C-r en parthe, tels que dans draf̌s, « drapeau » ${ }^{59}$, par exemple, qui n'existent plus en persan (diraf̌s). Il y a donc tout lieu de penser que l'ajout des voyelles d'anaptyxe est d'abord lié à un facteur interne.

\subsection{De la prothèse à l'anaptyxe}

La dernière phase a trait à l'évolution des voyelles de prothèse en voyelles d'anaptyxe dans les groupes consonantiques C-Occlusive. Constatons que le phénomène est plus large et englobe aussi les structures VCC- du moyen perse, avec des mots tels que $a \check{s} k a m b$, «ventre », ōšmurdan, « compter », uštar, « chameau », etc. Que la voyelle initiale soit étymologique ou prothétique, la question se pose de savoir si ce passage a eu lieu avec une phase intermédiaire, de nouveau avec présence de groupes consonantiques initiaux (VCC- > CC- > CVC-), ou si cette évolution est due à une interversion de la voyelle, qui passerait alors d'initiale à intérieure (VCC- > CVC-).

Pour Sādeghi ${ }^{60}$, l'apparition de la voyelle d'anaptyxe pourrait être une conséquence de la chute de la voyelle initiale, c'est-à-dire qu'il pose l'existence d'un stade où nous aurions eu de nouveau des groupes consonantiques initiaux. Voici le schéma qu'il propose pour le mot «chameau » : uštur > aštur ou ’̌stur > štur ou šztur. Étrangement il n'inscrit pas un štur dans son schéma alors que son raisonnement le suppose bel et bien. Pour aller dans son sens, on pourrait avancer comme argument l'existence de groupes consonantiques dans certains dialectes, - nous en avons vu le témoignage dans Šams-i Qais, ainsi que des exemples dans le Codex Cumanicus. Mais nous avions

\footnotetext{
${ }^{56}$ Cf. Paul 2005, p. 146.

${ }^{57}$ Notons néanmoins que Boyce (1977) et Durkin-Meisterernst (2004) présentent le mot sous une forme $d r \bar{o} \gamma$.

${ }^{58}$ Rappelons que selon Lentz le parthe pouvait aussi expliquer l'existence de $a b r \bar{u}(\S 3.1)$.

${ }^{59}$ Pour d'autres exemples, voir Boyce 1977 et Durkin-Meisterernst 2004.

60 Sādeghi 2001, p. 18. Par la suite, il discute cette théorie. Je souhaiterais apporter ici d'autres arguments.
} 
conclu que le phénomène datait d'une époque bien postérieure à l'apparition des voyelles d'anaptyxe, celles-ci étant attestées par des vocalisations dans les plus anciens manuscrits.

Quant à la chute de la voyelle initiale le problème est, selon moi, plus complexe que Sādeghi ne le présente ${ }^{61}$. Il met sur le même plan des mots où la chute de la voyelle ne

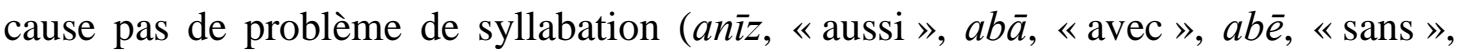
aya $\bar{a}$, «souvenir », etc.) et des mots dont la perte de la voyelle initiale provoquerait un nouveau groupe consonantique initial (aškamb, «ventre», uštar, «chameau», $\overline{o s ̌ m u r d a n, ~ « ~ c o m p t e r ~ », ~ e t c .) ~}{ }^{62}$. En outre, pour les mots où il est assuré qu'une voyelle initiale est tombée, il s'agit toujours d'une voyelle brève /a/. Comment alors s'explique la chute du /u/ de uštar, « chameau », et, surtout, de la voyelle longue / $\overline{0} /$ de ōšmurdan, «compter »? D'autre part, la voyelle initiale s'est maintenue en judéopersan dans 'br, « sur », 'by, «sans », 'b'z, « en arrière », etc. ${ }^{63}$ mais nous trouvons déjà škm, « ventre », šm'/šwm', « vous », šm'r, « compte », zw'n, « langue » (à comparer avec les mots moyen-perses aškamb, ašmāa, ōšmārag et uzwān). Il serait donc étrange qu'en judéo-persan, la voyelle initiale se soit maintenue dans les mots où elle pouvait tomber sans causer de problème de syllabation mais qu'elle soit tombée là où cette chute s'accompagnait d'un problème de prononciation avec de nouveaux groupes consonantiques initiaux. Qui plus est, ces mots apparaissent aussi dans le premier dialecte du Tafsīr d'Ezéchiel $\left(\mathrm{TE}_{1}\right)$, dialecte où nous savons que les groupes CC- étaient devenus CVC-. Il faudrait plutôt supposer que dans la seconde série de mots, la voyelle initiale n'est pas tombée mais qu'elle a été déplacée. Un dernier argument à l'encontre du lien entre chute de voyelles initiales et passage à la structure CVC doit être considéré : la date tardive où l'on trouve encore des occurrences du type uštur. On rencontre cette forme dans les Panj risāla-i târīxxi de Hāfiz-i Abrü ${ }^{64}$, texte du

\footnotetext{
${ }^{61}$ Certains /a/ initiaux se sont conservés jusqu'en persan contemporain, comme dans anār, « grenade », $a m \bar{\imath} r$, « prince », à côté de quelques occurrences de $n \bar{a} r$, mīr , dans les textes anciens (Cf. Lazard 1963, pp. 176-177, § 108-111).

62 Sādeghi 2001, p. 17. Quand il revient sur sa première hypothèse (p. 18), l'auteur ne mentionne pas cette différence essentielle.

${ }^{63}$ Lazard 1987a, pp. 168 et 172 ; Gindin 2007, III, Phonology, § 5.6. Le /a/ initial est aussi conservé dans le Qor'ān-e Qods (Lazard 1990, p. 186).

${ }^{64}$ Par exemple p. 66, 1. 19 de l'édition de Tauer (1959).
} 
$\mathrm{XV}^{\mathrm{e}}$ siècle, c'est-à-dire d'une époque où les formes comme abar ont disparu depuis longtemps ${ }^{65}$.

De plus, le persan comme le judéo-persan présentent déjà des voyelles d'anaptyxe dans les groupes C-r. Nous l'avons constaté par les vocalisations (en persan comme en judéo-persan du nord-ouest), par la métrique du persan et par les mater lectionis du judéo-persan, du moins le dialecte du nord-ouest $\left(\mathrm{TE}_{1}\right)$. Si tous les groupes $\mathrm{CC}-\mathrm{du}$ moyen perse tendaient à être brisés à cette époque, il serait étonnant que de nouveaux réapparaissent. Notons par ailleurs que cette apparition d'anaptyxe semble précéder la chute du /a/ initial : $\mathrm{TE}_{1}$ présente encore des voyelles initiales pour des mots comme abar, $a b \bar{a}, a b \bar{a} z, a b \bar{e}, a n \bar{z} z$ et déjà des voyelles d'anaptyxe dans les exemples cités plus

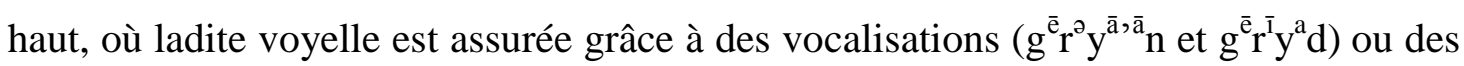
mater lectionis (bwrhn').

Enfin, pour les mots où la voyelle initiale est censée être tombée, nous avons des occurrences avec concurrence des deux formes VCC et CVC, dans des textes de la même époque, voire dans un même texte : pour le «ventre»par exemple, šikam (voyelle assurée avec la vocalisation šakam dans le Kitāb al-abniya) et ǐ̌kam dans le Tarjuma-i tafsìr-i Tabarī et le Kitāb al-mudxal ilā 'ilm ahkam al-nujūm de Abū Nasr Qummī $^{66}$. En judéo-persan, à défaut de vocalisation, nous avons des mater lectionis pour šwmwrdh ${ }^{67}$, «compté », et šwm', «vous » $(\mathrm{TE})^{68}$. Remarquons que tous ces termes ont en moyen perse la structure VCC (aškamb, ōšmurdan, ašmāa).

En revanche, il est possible que le passage à CVC soit lié à la chute des voyelles initiales : avant la chute totale, il peut y avoir eu changement du timbre vocalique en /ə/, mais sans l'étape $\mathrm{CC}: \mathrm{VCC}>{ }^{\mathrm{CCC}}>\mathrm{C} ə \mathrm{C}>\mathrm{CVC}$. Je rejoins ici le schéma que propose Sādeghi pour uštur. Nous pourrions déceler une trace de cet état intermédiaire avec une voyelle /a/, dans les vocalisations en shewa du judéo-persan et dans une

${ }^{65}$ Il apparaît comme une survivance dans les plus anciens textes et a disparu de la langue classique (Lazard 1963, p. 176, § 108).

${ }^{66}$ Voir d'autres occurrences dans Lazard 1963, p. 175, § 105.

${ }^{67}$ Asmussen 1968, p. 44. À moins que cela ne représente une voyelle longue (le mot vient du moyenperse ōšmurdan).

${ }^{68}$ Que nous ayons la graphie šm' dans EA ne remet pas en cause la présence de la voyelle. Il peut s'agir d'un usage graphique. Le second dialecte de TE, dont la langue est sensiblement la même que celle de EA, n'offre que des graphies šwm'. 
occurrence telle que ${ }^{\text {} ə \mathrm{a}} \mathrm{b}^{\mathrm{a}}{ }_{\mathrm{r}}{ }^{\mathrm{z}}$ (TE, 7, 11). Le copiste a ici indiqué une double vocalisation souscrite à l'alef initial : /o/ puis /a/. Étant donné que cette anomalie se situe dans un mot qu'il a en partie raturé, on peut imaginer que la première vocalisation correspondait au premier mot et la seconde au second : abar, « sur », et $a b \bar{a} z$, « en arrière ».

De ce qui vient d'être dit, il résulte que le passage par un nouveau groupe consonantique initial est exclu. Il nous faudrait alors envisager que le changement de VCC- à CVC- s'est produit par interversion du phonème vocalique. Sādeghi s'oppose à cette hypothèse en avançant que le timbre vocalique n'est pas toujours le même entre les deux structures ${ }^{69}$. Je ne pense pas qu'il y ait là un réel problème. Trois solutions sont en effet concevables : le timbre vocalique est passé par une phase de /a/; le timbre a changé quand cette voyelle s'est trouvée plus proche de la seconde voyelle du mot ; il y a eu analogie avec la voyelle d'anaptyxe la plus courante des groupes C-r, le /i/.

Souvenons-nous que le phénomène d'interversion existe bien entre le moyen perse et le persan pour briser les groupes consonantiques initiaux. Tous les mots débutant en moyen perse par fraC- commencent par farC- en persan (frabìh, «gras » > farbīh ; framān, « ordre » > farmān; frayād, « cri » > faryād ; frazand, « enfant » > farzand, etc.). L'interversion existe aussi dans drayāb, «mer»> daryā et dans driyōš, «pauvre » $>$ darvēes.

Si cette interversion de voyelle initiale à voyelle intérieure s'est produite, c'est probablement en raison d'une analogie. En comparant le nombre de mots moyenperses de structure C-Occlusive avec ceux du type C-r et C-w, c'est-à-dire ceux qui ont connu l'ajout d'une prothèse en moyen perse manichéen et ceux avec anaptyxe en persan, on s'aperçoit (Cf. tableau) que le second groupe est numériquement beaucoup plus important que le premier $(81 / 48)^{70}$. Et avec les mots moyen-perses VCC devenus CVC, la proportion ne change pas de manière significative (81/56).

\footnotetext{
${ }^{69}$ Sādeghi 2001, p. 17.

${ }^{70} \mathrm{Il}$ faudrait déterminer la fréquence de chaque vocable puisqu'il existe dans cette liste des mots très souvent utilisés et d'autres, plus rarement.
} 


\begin{tabular}{|l|l|}
\hline C-Occlusive & C-r/C-w \\
\hline 19 sp- (1 asp-) & 8 br- \\
16 st- & 18 dr- \\
9 šk- (1 ašk-) & 11 gr- \\
4 šn- (1 ašn- $)$ & 30 fr- \\
$(1$ ušt- $)$ & 8 sr- \\
$(1$ usk- $)$ & 4 xr- \\
$\left(1\right.$ ašm- $/ 1$ ǒs̆m- $\left.^{71}\right)$ & 1 tr- \\
$(1$ asw- $)$ & $1 \mathrm{dw}-$ \\
$(1$ uzw- $)$ & \\
\hline 48 (56) & 81 \\
\hline
\end{tabular}

L'analogie a donc fonctionné pour les mots VCC, que la voyelle ait été étymologique (présente dès le moyen perse) ou prothétique à partir du moyen perse manichéen. Dans le judéo-persan, on se rend compte que la première catégorie a même été plus souvent transformée que la seconde : on ne trouve plus que šs $(\mathrm{w}) \mathrm{m}$, « vous » (m.p. ašmā), šm'r, « compte » (m.p. ōšmārag), škmb, « ventre » (m.p. aškamb), zw’n, « langue » (m.p. uzwān), alors que 'šn's-, radical du présent de «connaître », alterne avec šn's- (nombreux exemples des deux dans EA) (m.p. šnāxtan/šnās-); stbr, « fort », avec 'stbr (m.p. stabr); 'zpwz-, «trahir », avec spwz- (m.p. spōxtan/spōz-). L'alternance peut parfois être d'ordre dialectal : pour les mots moyen-perses en sp-, le Tafsīr d'Ezéchiel (presque uniquement le premier dialecte) présente 'sp'h/'ysp'h, «armée » (m.p. spāh), 'spr, «bouclier » (m.p. spar), tandis que le texte grammatical karaïte (GK) a spyd, «blanc» (m.p. spēd). De même le premier dialecte du Tafsìr d'Ezéchiel a šnydn, «entendre », là où le second a exclusivement des formes 'kšnydn ${ }^{72}$ (m.p. ašnūdan). Notons ici que le dialecte du nord-ouest $\left(\mathrm{TE}_{1}\right)$ présente une voyelle de prothèse alors que le texte du sud-ouest GK n'en a pas ; et inversement la

\footnotetext{
${ }^{71}$ Je ne recense qu'une seule fois les mots de la même famille ōšmurdan et ōšmārag.

${ }^{72}$ Cette forme est surtout attestée dans les dialectes du sud : elle se retrouve dans le Qor'ān-e Qods (par exemple p. 12, 1. 9) et dans le Tārīx-i Sistān (Lazard 1963, p. 175, § 106).
} 
voyelle étymologique est préservée dans le dialecte du sud-ouest $\left(\mathrm{TE}_{2}\right)$. Il ne faudrait néanmoins pas en conclure que le judéo-persan du sud-ouest ignore les voyelles prothétiques. C'est ce que montrent les 'šn's déjà évoqués, ainsi que d'autres occurrences comme 'škn-, «briser » (m.p. šken-), par exemple en TE 187, 5, c'est-àdire dans le dialecte du sud-ouest du Tafsìr d'Ezéchiel $\left(\mathrm{TE}_{2}\right)$.

Les mots qui ont subi ce changement de VCC à CVC ont certes la structure VCOcclusive mais force est de constater que la nature de la première consonne joue également un rôle : ce sont les spirantes /s/, /š/ et /z/. Les mots avec Vmb- et Vrt- ne connaissent pas de transformation, malgré la tendance des voyelles initiales à tomber. Pour les groupes en VCC où la seconde consonne n'est pas une occlusive (Vbr-, Vbz-, Vrz-), il n'existe que de rares cas avec anaptyxe, encore ne s'agit-il que de variantes qui ne se sont pas maintenues en persan contemporain ${ }^{73}$. Certaines conservent le même timbre vocalique comme le /a/ dans barēšum, «soie », tandis que le /i/ plus courant de l'anaptyxe aurait été plus proche du /ê/ de la seconde syllabe. D'autres présentent un changement du timbre vocalique avec influence de la seconde voyelle

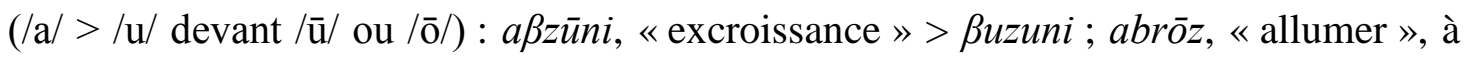
comparer avec burō $\gamma$, «éclat »; afzūn, « plus » > fuzūn.

Cette hypothèse permet d'expliquer ce qui pour Karimi ${ }^{74}$ paraît étrange : en persan contemporain, les mots d'emprunt débutant par $\mathrm{sC}$ - (en réalité C-Occlusive) connaissent l'ajout d'une voyelle de prothèse (esport, eski) alors que des mots d'origine persane ont le schéma sVC- (setāre, «étoile», surāx, «trou »). Les lois phonétiques n'étant valables qu'à une époque donnée, les groupes CVC ont exercé une influence sur les groupes VC-Occlusive dans le persan des premiers siècles, mais n'ont joué aucun rôle en persan contemporain.

\section{CONCLUSION}

Après avoir constaté la présence d'une voyelle d'anaptyxe dans le persan des premiers siècles, nous avons vu que l'hésitation, à première vue anarchique, entre voyelle de prothèse et voyelle d'anaptyxe, est liée à la nature des consonnes

\footnotetext{
${ }^{73}$ Lazard 1963 , pp. $175-176, \S 105$ et 106.

${ }^{74}$ Karimi 1987, p. 310.
} 
composant le groupe. Lorsqu'il a la structure C-r, la voyelle est insérée entre ces deux consonnes, ce qui donne un groupe CVC-; lorsque le groupe se présente en C-Occlusive, c'est alors soit une voyelle de prothèse, soit une voyelle d'anaptyxe qui est ajoutée. Ce constat a conduit à rechercher une raison historique à ce fait.

L'évolution des groupes consonantiques initiaux existant en moyen perse a eu lieu en trois phases (2). Dans un premier temps, dès le moyen perse manichéen, une voyelle de prothèse est venue briser les groupes $\mathrm{C}$-Occlusive. Dans le persan des $\mathrm{X}^{\mathrm{e}}$ $\mathrm{XI}^{\mathrm{e}}$ siècles, on découvre qu'un autre changement s'est déjà opéré : une voyelle d'anaptyxe s'insère dans les groupes C-r, sans que l'on puisse toutefois s'assurer que cette situation est aussi valable pour le judéo-persan du sud-ouest. Enfin, très probablement par analogie sur ces groupes devenus $\mathrm{CVC}$, les groupes $\mathrm{VCC}-$ que la voyelle ait été étymologique ou prothétique - se sont modifiés en structure CVC.

\section{(2) 1. C-Occlusive > VCC}

2. $\mathrm{C}-\mathrm{r}>\mathrm{CVC}$

\section{3. $\mathrm{VCC}>\mathrm{CVC}$}

Certains problèmes demeurent néanmoins. Le timbre de cette voyelle ajoutée semble obéir à des tendances et non à des règles strictes : tantôt /i/, tantôt /a/, tantôt /u/, ou bien il faut peut-être voir un premier timbre /ə/ qui aurait évolué. Par ailleurs, j'ai dû écarter les cas d'interversion puisqu'ils ne relevaient pas de la question des voyelles prothétiques ou anaptyctiques. Reste alors à connaître la raison pour laquelle la plupart des groupes consonantiques initiaux sont brisés par l'ajout d'une voyelle tandis que d'autres le sont par interversion, comme par exemple frazand devenu farzand. Tous les groupes moyen-perses fra- ont connu l'interversion, ainsi que quelques groupes dr-. Mais pourquoi les autres groupes en C-r ont ignoré cette solution de l'interversion? Pourquoi un mot comme brādar n'a pas évolué en bārdar? Aucune difficulté de syllabation ne s'y opposait pourtant. Enfin une analyse des proportions de cette hésitation entre anaptyxe et prothèse pour les groupes C-Occlusive est encore à mener si l'on veut saisir le rythme de la transformation et la force de l'analogie, mais 
avec un très grand nombre d'occurrences, ce que le cadre limité de cette étude ne m'a pas permis de réaliser.

Des problèmes demeurent, certes. Et pour les résoudre, ceux-là comme les autres, il faut se garder de la tentation de réduire les faits à

« des tableaux où les formes se rangent sagement au-dessous ou à côté les unes des autres $\gg^{75}$,

tentation de taire de possibles exceptions. Cette étude aura tenté de respecter la recommandation de Monsieur Lazard.

Bibliographie :

Asmussen J., 1965, Judaeo-persica II : The Jewish-Persian Law Report from Ahwāz, A.D. 1020, Acta Orientalia, XXIX, 1-2, p. 49-61.

Asmussen J., 1968, Jewish-Persian Texts. Introduction, Selection, and Glossary, Wiesbaden, Harrassowitz.

Bodrogligeti A., 1971, The Persian Vocabulary of the Codex Cumanicus, Budapest, Akadémiai Kiadó.

Boyce M., 1977, A word-list of Manichaean Middle Persian and Parthian, with a reverse index by Ronald Zwanziger, Leiden-Tehrān, Brill-Bibliothèque Pahlavi.

Durkin-Meisterernst D., 2004, Dictionary of Manichaean Texts. Vol. III, Part 1. Dictionary of Manichaean Middle Persian and Parthian, Turnhout, Brepols, Corpus Fontium Manichaeorum.

${ }^{75}$ Lazard 1987 b, p. 347. 
Firdaus̄̄, 1988-2007, Šāhnāma, Dj. Khāleghi-Motlagh, (ed), New York, Persian Heritage Foundation.

Gindin Th., 2007, The Early Judaeo-Persian Tafsìrs of Ezekiel. Vol. I : Text ; Vol. II : Translation; Vol. III : Commentary (non publié), Wien, Verlag der Österreichischen Akademie der Wissenschaften.

Hāfiz-i Abrū, 1959, Panj risāla-i tārīxī, F. Tauer (ed), Prague, Editions de l'Académie Tchécoslovaque des Sciences.

Karimi S., 1987, Farsi speakers and the initial consonant cluster in English, in G. Ioup \& S. H. Weinberger (eds), Interlanguage Phonology, New York-Cambridge, Newbury House, p. 305-315.

Khan G., 2000, Early Karaite Grammatical Texts, Atlanta, Society of Biblical Literature.

Lazard G., 1963, La langue des plus anciens monuments de la prose persane, Paris, Klincksieck.

Lazard G., 1974, Morphologie du verbe dans le parler persan du Sistan, Studia Iranica, 3/1, p. 65-85.

Lazard G., 1987a, Le judéo-persan ancien entre le pehlevi et le persan, in Societas Iranologica Europaea (ed), Transition periods in Iranian history, Actes du symposium de la Societas Iranologica Europaea, Fribourg-en-Brisgau, 22-24 mai 1985, ParisLeuven, Association pour l'Avancement des Études iraniennes-Peeters, p. 167-176.

Lazard G., 1987b, Catégories fonctionnelles et effets de sens : l'exemple de l'imparfait persan, in S. Mellet (ed), Études de linguistique générale et de linguistique latine offertes en hommage à Guy Serbat, Paris, Société pour l'information grammaticale, $\mathrm{p}$. 347-354.

Lazard G., 1990, Lumières nouvelles sur la formation de la langue persane : une traduction du Coran en persan dialectal et ses affinités avec le judéo-persan, in Sh. Shaked \& A. Netzer (eds), Irano-Judaica II, Studies Relating to Jewish Contacts with Persian Culture Throughout the Ages, Jérusalem, Ben-Zvi Institute, p.184-198.

Lazard G., 2002, Encore la versification Pehlevie, Jerusalem Studies in Arabic and Islam, 26, p. 130-139. 
Lazard G., 2003, Du pehlevi au persan : diachronie ou diatopie ?, in L. Paul (ed), Persian Origins : Early Judaeo-Persian and the Emergence of New Persian. Collected papers of the Symposium, Göttingen, 1999, Wiesbaden, Harrassowitz, p. 95-102.

Lazard G., 2006, Grammaire du persan contemporain, avec la collaboration de Y. Richard, R. Hechmati et P. Samvelian, Tehrān, IFRI-Farhang Mo'āser, nouvelle édition.

Lazard G., 2009, Qu'est devenue la préposition $\bar{o}$, in E. Pirart \& X. Tremblay (eds), Zarathushtra entre l'Inde et l'Iran: études indo-iraniennes et indo-européennes offertes à Jean Kellens à l'occasion de son $65^{e}$ anniversaire, Wiesbaden, Ludwig Reichert, p. 169-176.

Lazard G., à paraître, La dialectologie du persan préclassique à la lumière des nouvelles données judéo-persanes, Actes du colloque de Hambourg (Irano-Judaica VI), septembre 2006.

Lentz W., 1926, Die nordiranischen Elemente in der neupersischen Literatursprache bei Firdosi, Zeitschrift für Indologie und Iranistik, 4, p. 251-316.

MacKenzie D. N., 1968, An early Jewish-Persian argument, Bulletin of the School of Oriental and African Studies, 31/2, p. 249-269.

MacKenzie D. N., 1970, A concise Pahlavi dictionary, London, Oxford University Press.

MacKenzie D. N., 1979-1980, Mani’s Šābuhragān, Bulletin of the School of Oriental and African Studies, 42, p. 500-535 et 43, p. 288-310.

Moinfar Dj., 1973, Phonologie quantitative du persan d'après le Livre des Rois de Firdausī, Paris, Dunod.

Paul L., 2002, Grammatical and Philological Studies on the Early Judaeo-Persian Texts from the Cairo Geniza, thèse d'habilitation non publiée, Göttingen, p. 202-254 (Texts).

Paul L., 2003, Early Judaeo-Persian in a Historical Perspective: The Case of the Prepositions $b e, u, p a(d)$, and the Suffix $r \bar{a}$, in L. Paul (ed), Persian Origins : Early Judaeo-Persian and the Emergence of New Persian. Collected papers of the Symposium, Göttingen, 1999, Wiesbaden, Harrassowitz, p. 177-194. 
Paul L., 2005, The Language of the Šāhnāme in Historical and Dialectal perspective, in D. Weber (ed), Languages of Iran : Past and Present. Iranian Studies in memoriam David Neil MacKenzie, Wiesbaden, Harrassowitz, p. 141-151.

Pisowicz A., 1985, Origins of the New and Middle Persian phonological systems, Krakow, Uniwersytet Jagiellonski.

Ravāqi, 'A., 1364/1985, Qor'ān-e Qods, kohantarin bargardān-e Qor'ān be fārsi, Tehrān, Mo'assese-ye farhangi-e Mohammad Ravāqi.

Sādeghi ‘A. A., 1349/1970, Az bein raftan-e goruh-e sāmet-e āqāzi, Soxan, 20/6, p. 543550.

Sādeghi ‘A. A., 1380/2001, Tahavvol-e xuše-ye sāmet-e āqāzi dar zabān-e fārsi, Nāme-ye Irān-e bāstān (The International Journal of Ancient Iranian Studies), 1/1, p. 15-24.

Thomason S., 2001, Contact-induced typological change, in M. Haspelmath, E. König, W. Österreicher \& W. Raible (eds), Language Typology and Language Universals. Sprachtypologie und sprachliche Universalien. La typologie des langues et les universaux linguistiques, Berlin-New York, Walter de Gruyter, p. 1640-1648.

Utas B., 1969, The Jewish-Persian Fragment from Dandān-Uiliq, Orientalia Suecana, 17, p. $123-136$. 